\title{
Design and Develop Real Estate Information System Based on Map World API
}

\author{
He Bi \\ College of Civil Engineering, Shandong Jiaotong University, Jinan, China \\ hebiy@163.com
}

Keywords: Information System; Real Estate; Map World API

\begin{abstract}
To improve the management level of real estate enterprises, and determine the development of the real estate information management system, this paper, using Map World API as the base of spatial data, calls the API in JavaScript, and inputs attribute data through programming in IDE. After the set of data sets is completed, various functions was realized through the function in API. At the end, through network configuration, the real estate information system has been established.
\end{abstract}

\section{Introduction}

Real estate industry has become a very important industry in China, its value has reached 82 trillion in 2017. With the growth of real estate prices, real estate transactions have become one of the most popular transactions in the market. Cause the volume and number of transactions has increased dramatically, management of relevant information become more and more complicated. It is very necessary to develop an information system to manage these information. Real estate information is closely related to spatial information and attribute information, so it is more appropriate to develop the system with GIS. For develop GIS, the cost of obtaining basic geographic data is very high, and Map World API can provide the basic geographic data. Therefore, it is very appropriate to develop this system based on Map World API.

\section{Map World API}

"Map World" is China national geographic information public service platform, is the network geographic information sharing and service portal built by the national basic geographic information center. It integrates the geographic information departments at all levels from the state, provincial and municipal, as well as the government departments, enterprises, institutions and society. The geographic information public service resources of the group and the public provide authoritative, standard and unified online geographic information integrated services to all kinds of users.

Map World API 4.0 for JavaScript is a set of application interfaces that conform to the HTML5.0 specification, providing various map services and data in the form of HTML5.0, such as map display, annotation, location, and so on. It provides the developers a quick call to call the map online geographic information services, including quickly creating maps, calling maps, POI 
(Position Of Interesting) search, and adding coverage on the map. It can help developers build rich and interactive map applications in the website, support PC and mobile browser based map application and development, and su pport map development of the mainstream HTML4.0 and HTML5.0 features.

Cause these characteristics and functions, Map World API has become an excellent choice for developing real estate information system.

\section{System Design}

System design is the first step of system development. The task of system design is to determine the structure of the system, the development environment and the functions of each module, it is the guidance of the whole development work. In this research, the structure of the system was designed as Figure 1.

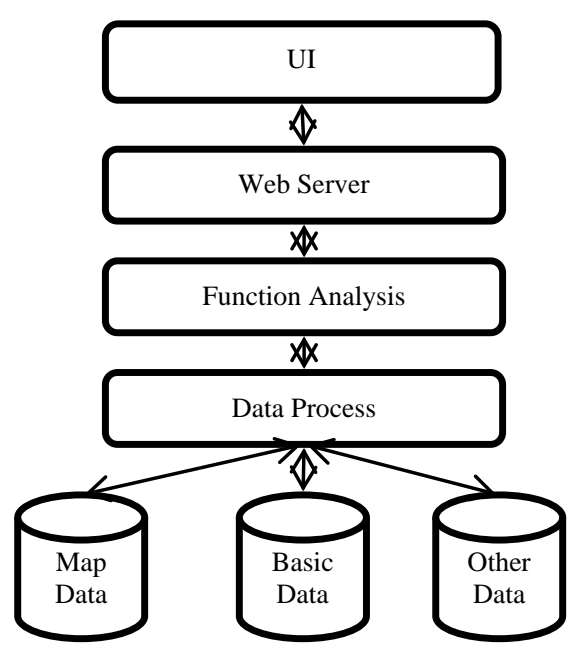

Figure 1. System Structure

The data of the system consists of three parts: map data, basic data and other data. Map data mainly refers to spatial data, which is used to express geographic location information of objects, basic data mainly refers to attribute data, which is used to express attribute of objects, just like name, area, owner, other data refers to other data which the system needs, just like user name and password. The management of all these data is done centrally by the data analysis module.

Function analysis module is the core of the system. It is made up of a series of functional functions, and its task is to complete the tasks assigned by the user, to complete these tasks by calling data and function functions, and to return the processing results to the user.

Web server is a bridge between the client and the server, which transfers the user's commands to the server, and also transfers the results of the server execution to the users.

UI (User Interface) is the operation interface provided by the system to users, users browse information on UI, issue commands and use the system.

The development tool of the system is Dreamweaver, and the development language is JavaScript. The reason for using Dreamweaver as development tool is that it can develop a beautiful user interface with CSS and other tools, and can easily use JavaScript to develop application functions.

\section{Call API}

The first step of the system implementation is to call Map World API. Map World API provides a variety method of calling, it can be called in web pages and mobile devices. In this research, it was 
called in web pages, the code in JavaScript is:

$<$ !DOCTYPE html $>$

$<$ html $>$

$<$ head $>$

$<$ meta charset="UTF-8"/>

$<$ title $>$ REIS $</$ title $>$

$<$ script type $=$

"text/javascript" src="http://api.tianditu.com/api?v=4.0" $></$ script $>$

$<$ script $>$

var map;

var zoom $=12$;

function onLoad() \{

map = new T.Map('mapDiv');

map.centerAndZoom(new T.LngLat(116.40769, 39.89945), zoom);

\}

$</$ script $>$

$</$ head $>$

$<$ body onLoad="onLoad()">

$<$ div id="mapDiv" style="position:absolute;width:500px; height:400px" $></$ div $>$

$</$ body $>$

$</$ html $>$

The code include six step: prepare web page, insert Map World API document, create a map container, create a map instance, determine the latitude and longitude coordinates, map initialization.

Through these works, the basic geographic information data provided by Map World was loaded into the system, and users can browse the corresponding map on the client side.

\section{Data Input}

After calling Map World API in the system, the basic geographic information has been loaded, but, the real estate information has not been loaded, so the next step is real estate data input.

In order to organize real estate data, each real estate target is regarded as an object. The data of every object include two parts: spatial data and attribute data. Spatial data represent location information of real estate objects, through spatial data, customs can get where are the real estate object. Attribute data represent how are the real estate object, just like the price, area, age, etc. Combining these two parts of data, users can have a detailed understanding of real estate object, then they can choose the appropriate house or apartment or other object.

The spatial data can be input by the following code:

$<$ script $>$

var map;

var zoom = 12;

function onLoad() \{

map = new T.Map("mapDiv");

map.centerAndZoom(new T.LngLat(117.6573, 38.5672), zoom);

var marker $=$

new T.Marker(new T.LngLat(117.4117, 38.9068));

map.addOverLay(marker);

\}

$</$ script $>$ 
The attribute data can be input by the following code:

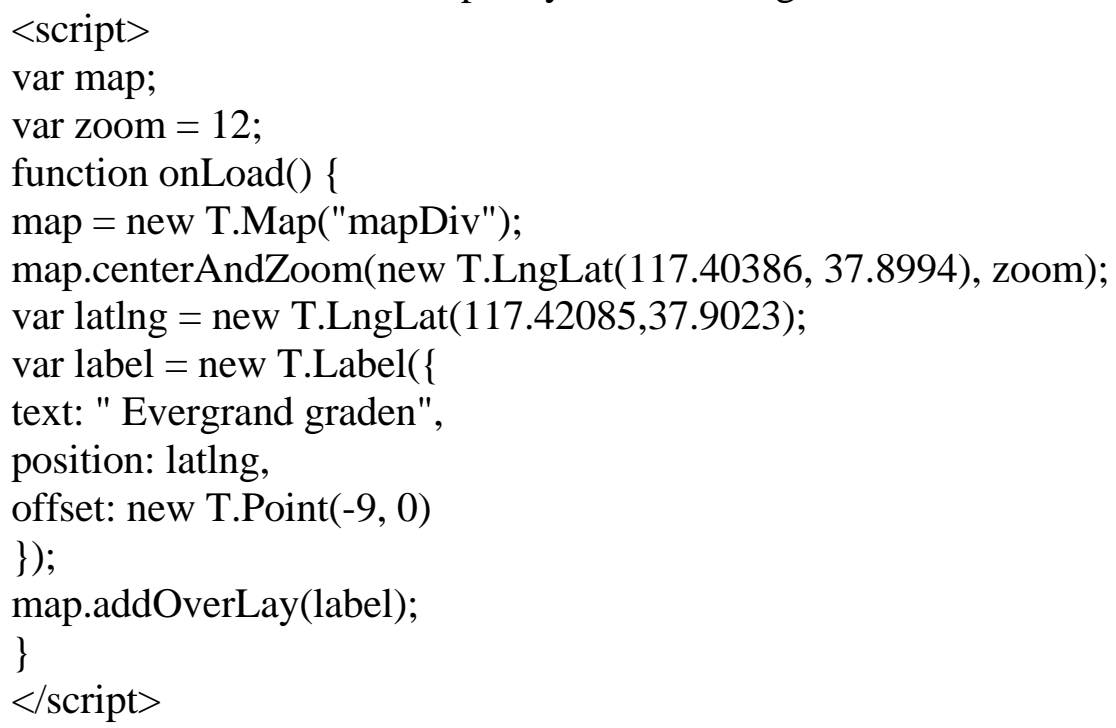

If the object is more characteristic, for example, the area is very large and can not be expressed in the form of points, it can be expressed in the form of a surface, If the object expressed as a surface, it can be implemented by the following code:

$<$ script $>$

var map;

var zoom = 12;

function onLoad() \{

map = new T.Map("mapDiv");

map.centerAndZoom(new T.LngLat(116.40969, 39.94940), zoom);

var points $=[]$;

points.push(new T.LngLat(116.41136, 39.97569));

points.push(new T.LngLat(116.411794, 39.9068));

points.push(new T.LngLat(116.32969, 39.92940));

points.push(new T.LngLat(116.385438, 39.906));

var polygon $=$ new T.Polygon $($ points, \{

color: "blue", weight: 3, opacity: 0.5, fillColor: "\#FFFFFF", fillOpacity: 0.5

\}$)$

map.addOverLay(polygon);

\}

$</$ script $>$

Through data input operation, we can input all data of real estate objects into the system on the basis of basic spatial data, with these data, the other function can be realized.

\section{Function Realization}

\subsection{Map Browsing and Operation}

Compared with the common management information system, the biggest advantage of GIS is that it can visually enable users to get information in the form of map. After calling Map World API and data input operation, all the data of real estate information system can be expressed in one map. In order to enable users to better use this map, we need to design map browsing and operation functions. These functions include magnification, reduction, translation, rotation, distance measurement, area measurement and eagle eye function. Map World API provides these functions, 
which only need to call corresponding functions when developing the system, and these functions will have corresponding general buttons placed on the user interface for users to use conveniently. Here is an example of the code of the hawk eye function:

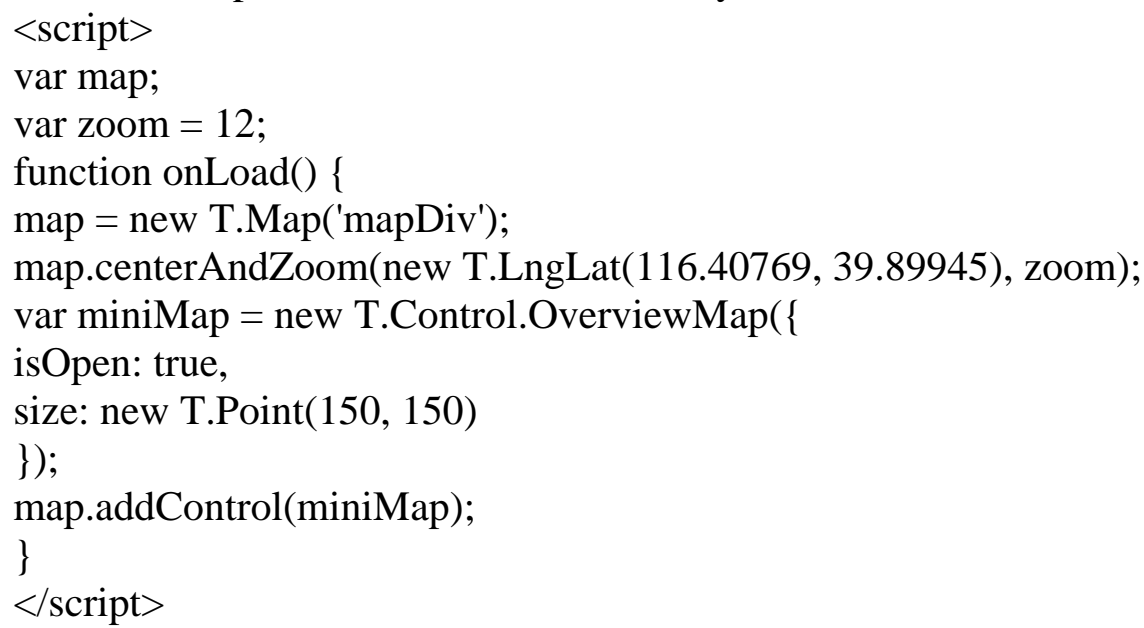

\subsection{Attribute Query}

Attribute query means that when the user wants to view the attribute information of an real estate object, they can click the object on the user interface, and the system can query the information of the object and display it to the user. This function can be realized by the following code:

$<$ script $>$

var map, pLngLat;

var zoom = 15;

function onLoad() \{

var sContent = "Evergrand garden, $135 \mathrm{~m}^{2}$ ";

map = new T.Map("mapDiv");

pLngLat $=$

new T.LngLat(117.5812,37.4788);

map.centerAndZoom(pLngLat, zoom);

var infoWindow = new T.InfoWindow(sContent);

map.openInfoWindow(infoWindow, pLngLat); //

document.getElementById("result").innerHTML $\quad=\quad$ "Information: $<\mathrm{br} \quad />\quad \quad+$ infoWindow.getContent();

\}

$</$ script $>$

\subsection{0bject Query}

Object query means that when customers provide conditions for the real estate object they want, just like name, location, area, price, the system can query out the joint object by these conditions, and display them on the screen for customers. This function can be realized by the following code:

$<$ script>

var map;

var zoom = 12;

function onLoad() \{

map = new T.Map("mapDiv");

map.centerAndZoom 
(new T.LngLat(117.40969, 37.89945), zoom);

var lo = new T.Geolocation();

$\mathrm{fn}=$ function (e) \{

if (this.getStatus ()$==0)\{$

map.centerAndZoom(e.lnglat, 15)

alert("getcod:

"+e.lnglat.lat + "," + e.lnglat.lng)

var marker $=$ new T.Marker(e.lnglat);

map.addOverLay(marker);

\}

if(this.getStatus ()$==1)\{$

map.centerAndZoom(e.lnglat, e.level)

alert("getcode:

"+e.lnglat.lat + "," + e.lnglat.lng)

var marker = new T.Marker(e.lnglat);

map.addOverLay(marker);

\}

\}

lo.getCurrentPosition(fn);

\}

$<$ script $>$

\subsection{Business Information Storage}

When real estate transactions completed, the business information should be stored in the system caused these information are associated to the status of real estate object. In this system, these information were stored in MySQL database, data table was created for storing these information, and main framework connects the database through jQuery.

\section{Conclusion}

The establishment of information system is an effective way to improve business efficiency. This research aims at the demand of real estate business, established a management information system based on GIS by using Map World API, realized collection, storage and management of spatial data and attribute data. On this basis, just like map and information browsing, position and property query, all functions of real estate management information system has been realized. This proved that using Map World API to develop real estate information system is an effective technical path.

\section{Acknowledgment}

This work was financially supported by Shandong Jiaotong University Science Fund (Z201613) and Shandong province transportation development Fund.

\section{References}

[1] Li. Jiansong, TANG. Xuehua, “The Principle of Geographic Information System," Wuhan: Wuhan University Publication, 2015, pp. 12-16.

[2] He. Bi, LI Haitao, SUN Gengxin, “The Principle of Geographic Information System,” Peking: TSinghua University Publication, 2010, pp. 5-12.

[3] Zhang Jingxiong, “Geographic Information System and Science,” Wuhan: Wuhan University Publication, 2010, pp. 
92-99.

[4] Qin Kun, "Theories and Methods of Spatial Analysis in GIS," Wuhan: Wuhan University Publication, 2011, pp. 122-128.

[5] Kang-tsung Chang, "Introduction to Geographic Information System," Peking: Science Press, 2018, pp. 156-168.

[6] Wang Yang, Jin Lixia Zhang Hong'ou, "The Spatial Differentiation Pattern and Formation Mechanism of Housing Condition in Guangzhou City, China," Scientia Geographica Sinica, vol. 37, Jun. 2017, pp. 868-875, doi:10.13249/sgs.2017.06.008.

[7] Wu Danxian and Zhou Suhong, “The Matching Pattern of Housing-Shopping Space Based on Residents' Daily Shopping Behaviors: A Case Study of Communities in Guangzhou" Scientia Geographica Sinica, vol. 37, Feb.2017, pp. 229-235, doi:10.13249/sgs.2017.02.08. 